Jonel Donn Leo S. Gloria, MD'

'Department of Otorhinolaryngology Philippine General Hospital University of the Philippines Manila Philippines
Correspondence: Dr. Jonel Donn Leo S. Gloria

Department of Otorhinolaryngology

Ward 10, Philippine General Hospital

University of the Philippines Manila

Taft Avenue, Ermita, Manila 1000

Philippines

Phone: (632) 554-8400 local 2151

E-mail: jonel.gloria@gmail.com

Reprints will not be available from the author.

The author declared that this represents original material that is not being considered for publication or has not been published or accepted for publication elsewhere in full or in part, in print or electronic media; that the manuscript has been read and approved by the author, that the requirements for authorship have been met by the author, and that the author believes that the manuscript represents honest work.

Disclosures: The author signed a disclosure that there are no financial or other (including personal) relationships, intellectual passion, political or religious beliefs, and institutional affiliations that might lead to a conflict of interest.

Presented at the Annual Residents' Interesting Case Contest (1st place), Philippine General Hospital - Department of Otorhinolaryngology, Manila, February 24, 2012.

\title{
Extragnathic Sinonasal Ameloblastoma: A Rare Benign Intranasal Tumor with Malignant Features
}

\section{ABSTRACT}

Objective: To report a case of extragnathic sinonasal ameloblastoma and discuss its clinical features, approach to diagnosis, pathology and management.

Methods:

Design: Case Report

Setting: Tertiary Government UniversityHospital

Patient: One

Results: A 40-year-old f emale consulted for a rapidly enlarging right intranasal mass of four months duration associated with recurrent profuse epistaxis and nasal obstruction. Previous specimens of the mass were histopathologically interpreted as ameloblastoma versus craniopharyngioma. Examination revealed a pink, fleshy, smooth right intranasal mass with associated nasomaxillary bulge and supero-lateral displacement of the right eye. Computed tomography (CT) scan and magnetic resonance imaging (MRI) of the nasal cavity and paranasal sinuses demonstrated a soft-tissue density occupying the entire nasal cavity with erosion but no invasion of the maxillary sinus and no intracranial extension despite erosion of the skull base. The mass was completely excised via lateral rhinotomy and the final histopathologic diagnosis was ameloblastoma.

Conclusion: Extragnathic sinonasal ameloblastoma is a benign but locally aggressive variant of ameloblastoma involving the nasal cavity and/or paranasal sinuses often mimicking malignant tumors. Diagnosis is primarily based on histopathology but radiologic and intraoperative findings help distinguish it from differentials. Complete surgical excision remains the treatment of choice, and coupled with good follow up, may improve the prognosis of patients.

Keywords: sinonasal ameloblastoma, extragnathic, craniopharyngioma

Signs and symptoms of a recurrent rapidly enlarging intranasal mass, epistaxis, nasal obstruction and displacement of the ipsilateral globe lead one to suspect a possible malignancy. A physician who performs a biopsy may be surprised by a histopathologic diagnosis of 
ameloblastoma - not only because it is benign but also due to its unusual location in the nasal cavity (being odontogenic). Although benign, ameloblastoma is a locally aggressive tumor predominantly involving tooth-bearing regions of the oral cavity including the mandible and the maxilla. It is relatively rare comprising only $1 \%$ of all head and neck tumors despite being the most common true odontogenic neoplasm with an incidence of $11 \%$.

Extragnathic ameloblastoma is a variant of ameloblastoma that appears to elude its pathogenesis as it arises outside the boundaries of the odontogenic apparatus. ${ }^{2}$ Extragnathic ameloblastoma primarily from the nasal cavity is extremely rare with only few documented reports in the literature. ${ }^{2-6}$ Its unusual location and highly aggressive behavior make it a worthy consideration among the differential diagnosis of nasal masses that should be of interest not only to ENT surgeons with special interest in rhinology, but to maxillofacial surgeons, oral surgeons and pathologists.

Here is one such case of extragnathic sinonasal ameloblastoma and a discussion of its clinical features, approach to diagnosis, pathology and management.

\section{CASE REPORT}

A 40-year old female presented with a 4-month history of persistent mucoid, non-foul smelling, occasionally blood-tinged right rhinorrhea and recurrent nasal congestion. A previous intranasal mass punch biopsy by an otorhinolaryngologist revealed ameloblastoma; the final histopathologic diagnosis following undisclosed nasal surgery by another otorhinolaryngologist was craniopharyngioma. One month after surgery, the patient experienced recurrence of nasal obstruction and rhinorrhea, with bulging of the right nasal bridge, an enlarging right intranasal mass and spontaneous recurrent profuse epistaxis. This prompted emergency consult at our hospital.

On examination a right nasomaxillary bulge and supero-lateral displacement of the right eye were evident. Nasal endoscopy revealed a pink, fleshy, smooth mass with foul-smelling, mucoid discharge in the right nasal vestibule and contralateral septal deviation. The nasopharyngeal mass was appreciated on posterior rhinoscopy. The rest of the head and neck examination findings were unremarkable. Plain and contrast-enhanced axial and coronal computed tomography images of the nasal cavity and paranasal sinuses showed a large, lobulated, heterogeneously enhancing intranasal mass measuring $4.15 \times 4.95 \times 8.01 \mathrm{~cm}$ (transverse, craniocaudal, anteroposterior) occupying the entire nasal cavity. (Figures 1 and 2) The mass extended to the ethmoid sinuses with suspicious extension into the right orbit, sphenoid sinus, nasopharynx and partially to the right maxillary sinus with associated thinning of the medial maxillary wall.

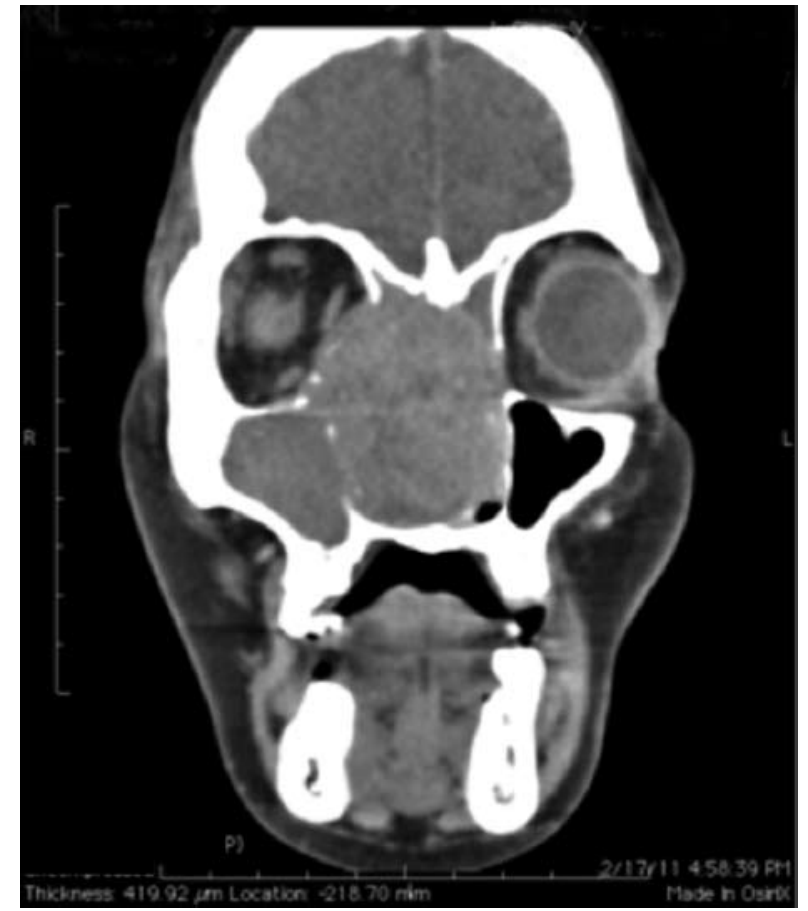

Figure 1. Contrast-enhanced CT scan of the nasal cavity and paranasal sinuses, coronal view showing a heterogeneously enhancing mass occupying the entire nasal cavity with thinning of the right medial maxillary wall and lamina papyracea. There is opacification of the right maxillary sinus.

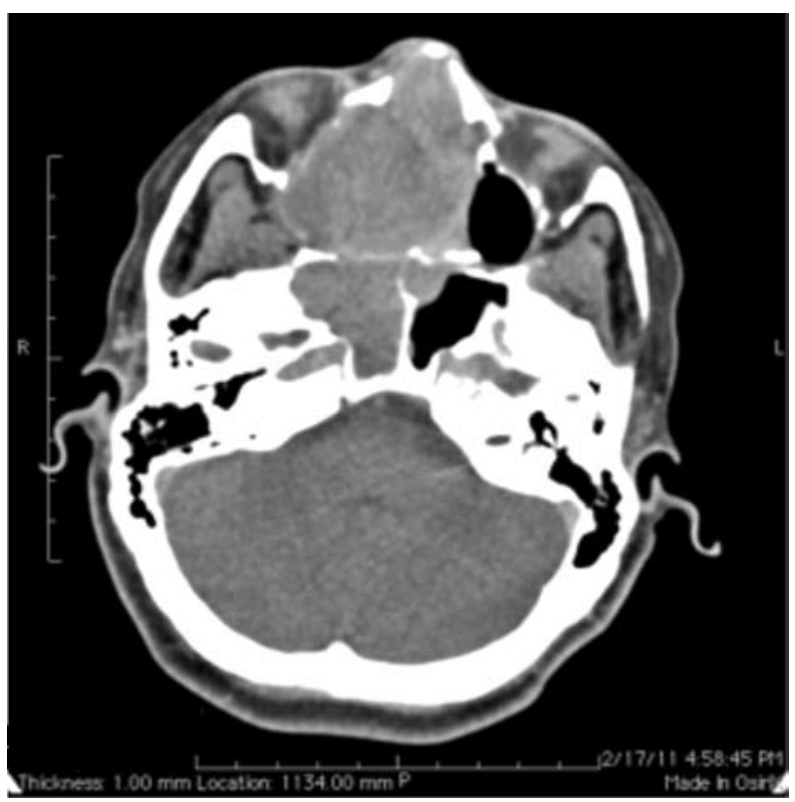

Figure 2. Contrast-enhanced CT scan of the nasal cavity and paranasal sinuses, axial view showing additional opacification of the right sphenoid sinus.

Due to the extensive involvement of adjacent structures, MRI was requested to rule out intracranial extension or origin (excluding craniopharyngioma) and paranasal origin (excluding gnathic maxillary ameloblastoma) of the tumor. MRI revealed fluid accumulation-possibly from obstruction without invasion of the maxillary sinus. 
CASE REPORTS

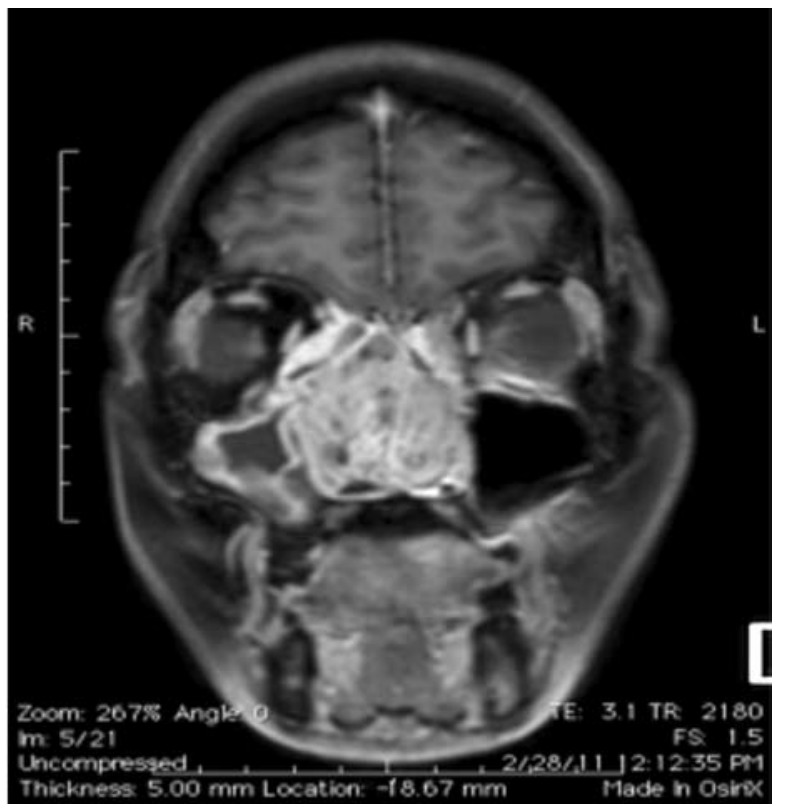

Figure 3. MRI of the nasal cavity and paranasal sinuses with gadolinium, coronal view showing a heterogeneously contrast-enhancing mass in the nasal cavities with fluid accumulation in the right maxillary sinus.

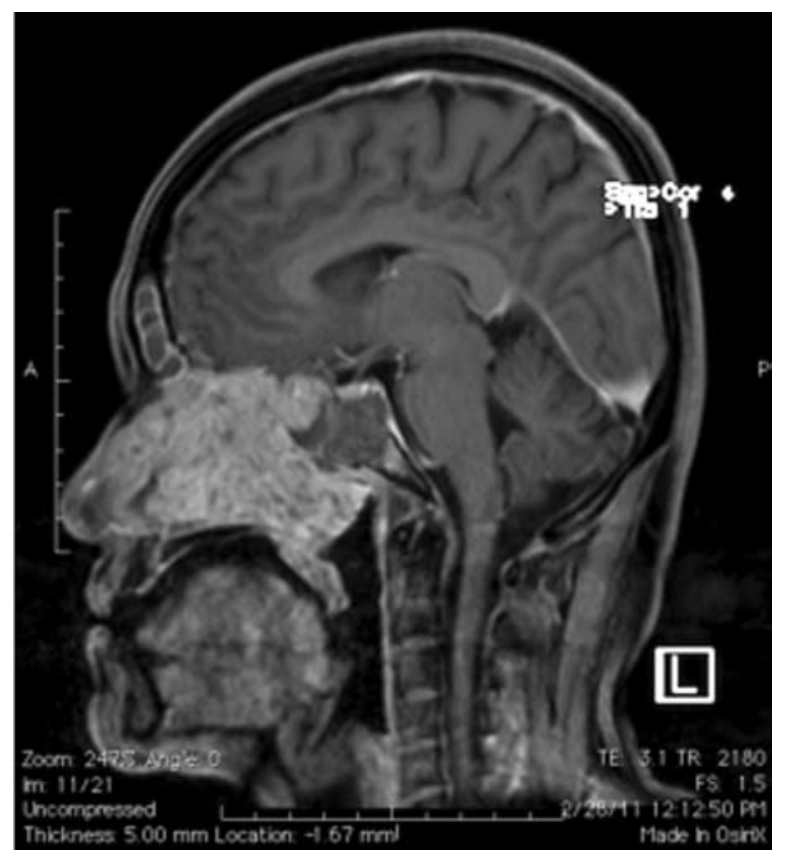

Figure 4. MRI of the nasal cavity and paranasal sinuses with gadolinium, sagittal view showing a heterogeneously contrast-enhancing mass in the nasal cavities with extension to the right sphenoid sinus.

(Figure 3) The sphenoid sinus was only partially occupied by the tumor alongside fluid accumulation. (Figure 4) Likewise, there was no evidence of intracranial extension or origin.

A slide review of the specimen from the previous surgery was interpreted as consistent with ameloblastoma versus

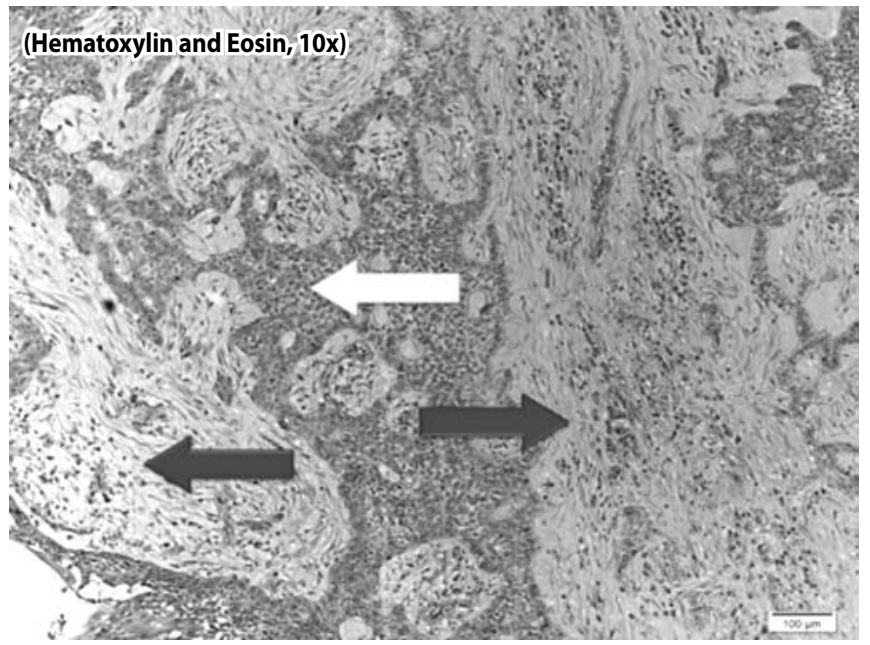

Figure 5. Histopathologic slide, Hematoxylin and Eosin stain, scanning view (10x) shows blue-gray relatively less cellular areas (blue arrow) and dark blue cellular areas (yellow arrow). The blue-gray areas correspond to the loose, fibrous stroma while the dark blue cellular areas correspond to the interspersed cords and islands of epithelial cells.

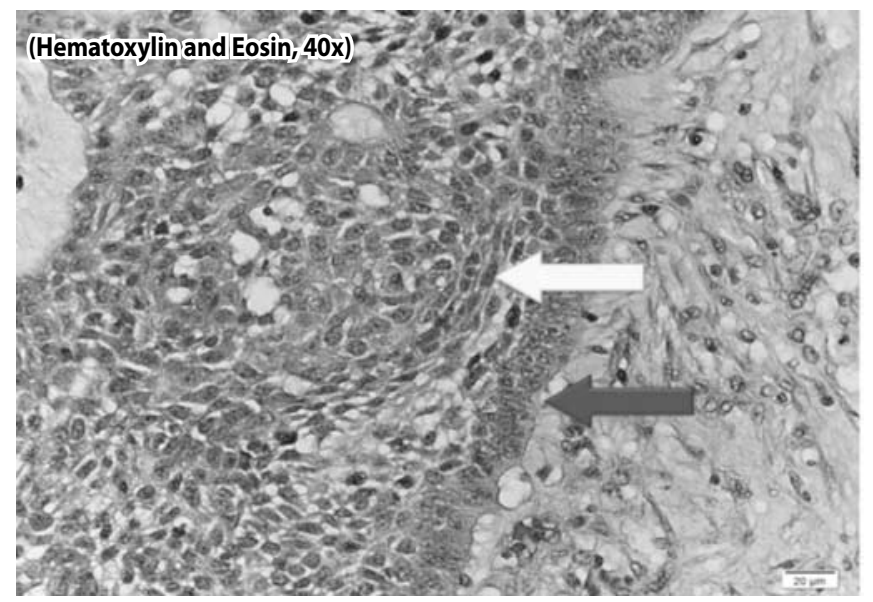

Figure 6. Histopathologic section, Hematoxylin and Eosin stain, higher magnification (40x) of the epithelial component shows two cell types. In the periphery, there are columnar cells that show palisading with a reversed polarity arrangement (dark arrow). Medial to it, the cells are arranged more loosely, corresponding to the stellate reticulum. Higher magnification of the stroma (light arrow) shows fibroblasts arranged in loose connective tissue.

craniopharyngioma. Excision of the mass via right lateral rhinotomy and partial medial maxillectomy yielded a grayish, friable, fungating mass occupying the right nasal cavity attached to the right posteromedial choana extending to the sphenoid sinus pushing against (but not involving) the septum with no involvement of the right maxillary sinus or attachment to the skull base. Final histopathology was signed out as ameloblastoma. No recurrence was noted after 11 months of postoperative follow up.

\section{DISCUSSION}

Ameloblastoma is a locally aggressive benign tumor of odontogenic tissues with a high rate of recurrence if not adequately excised. It represents $1 \%$ of all oral cavity tumors, generally appearing in the 


\section{CASE REPORTS}

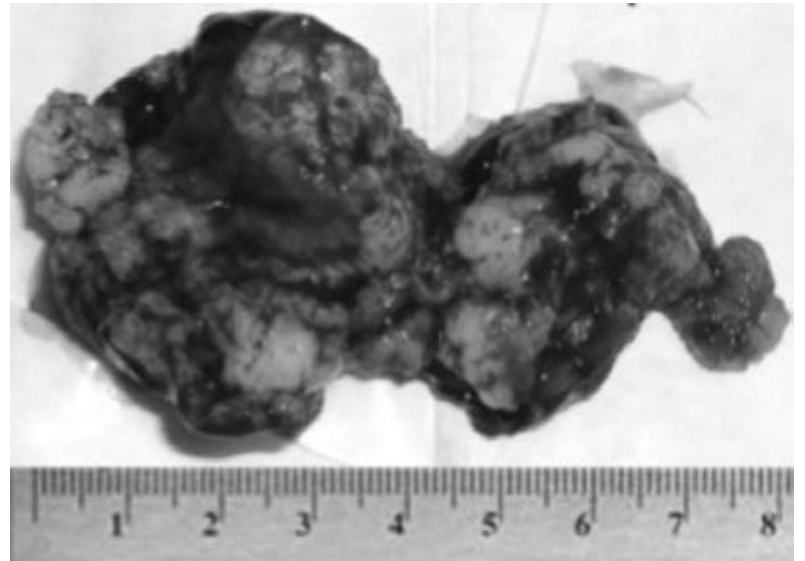

Figure 7. Gross specimen consisting of cream tan, irregular, soft to rubbery tissue fragments with an aggregate diameter of $8 \mathrm{~cm}$.

mandible in $80 \%$ of cases and $15-20 \%$ in the maxilla. ${ }^{2}$

Differential diagnoses of an intranasal ameloblastoma include 1) gnathic ameloblastoma from the maxilla with the nasal cavity only secondarily involved; 2) extragnathic ameloblastoma, a variant of ameloblastoma that arises primarily in the sinonasal mucosa known as primarysinonasalameloblastoma ${ }^{7}$; and 3) infrasellarcraniopharyngioma extending down to the nasal cavity. The majority of intranasal ameloblastomas in the literature are actually of maxillary origin with extension into the nasal cavity. ${ }^{8}$ Ameloblastoma exclusively arising in the sinonasal tract is extremely rare, with very few reported cases in the literature ${ }^{2-6}$ and its presence should be well established before making a definitive diagnosis. There are even fewer reports of infrasellar craniopharyngioma with intranasal extension in the literature., ${ }^{9,10}$ The difficulty in distinguishing these two entities (ameloblastoma and craniopharyngioma) as evidenced by the histopathologic reports in this case stems from the very similar histopathologic features reflective of their odontogenic origins. In addition, the possibility of malignant ameloblastic carcinoma (although rare) should never be discounted, especially in aggressive recurrent cases ${ }^{11}$ nor should malignant transformation of ameloblastoma be overlooked (diagnosed with specific staining like CK AE1/AE3). ${ }^{12}$

The histopathologic sections (Figures 5 and 6 ) showing odontogenic epithelium arranged in long strands and cords that appear to surround central areas of supporting stroma with palisading columnar cells exhibiting reverse polarization surrounding loosely arranged stellate reticulum-like epithelium are compatible with a diagnosis of ameloblastoma, both for gnathic and extragnathic variants. Although the same features are found in craniopharyngioma, the absence of cystic formation, degenerative changes, calcifications and cholesterol clefts $^{6}$ characteristic of the latter favor a diagnosis of ameloblastoma.
As evidenced by the histomorphologic, radiologic and intraoperative findings in the patient, a diagnosis of extragnathic sinonasal ameloblastoma was established.

Extragnathic ameloblastomas comprise only $2-10 \%$ of all ameloblastomas. ${ }^{10}$ Extragnathic sinonasal ameloblastomas are even less common. Schafer et al. reviewed nearly 20,000 sinonasal tumors over a 40-year period and reported only 24 cases of ameloblastoma exclusively arising in the sinonasal tract. ${ }^{8}$ To date, only five additional case reports have been published, based on a PubMed and Google search using the keywords "ameloblastoma," "sinonasal," and "extragnathic."2-6 The overall mean age at presentation is 59.7 years and more males are affected than females with a ratio of $3.8: 1 .{ }^{4}$ In contrast, this case involved a relatively young 40-year-old female.

Usual presenting signs and symptoms mimic those of malignant tumors which include intranasal mass, nasal obstruction, sinusitis, epistaxis, facial swelling, dizziness, and headache. ${ }^{8}$ In this case, the intranasal mass, epistaxis and nasal obstruction are consistent with the usual signs and symptoms. The additional supero-lateral displacement of the right globe can be attributed to tumor mass effect.

Sinonasal amelobastomas are described as polypoid, predominantly solid masses with glistening gray-white, pink or yellow-tan color, ranging from a few millimeters to $9.0 \mathrm{~cm}$ with consistency varying from rubbery to granular ${ }^{8}$ consistent with the cream to tan, fleshy, rubbery mass in this case. (Figure 7)

CT scans of the nasal cavity and PNS were the primary imaging modalityin previous reports. Theappearanceofsinonasalameloblastoma depends on tumor extent, generally as a solid mass, soft tissue density or opacification occupying the nasal cavity and/or the paranasal sinuses with occasional bony erosion as seen in this case.,8 The additional use of MRI in this case was beneficial in delineating tumor extent.

The reported sites of origin of primary sinonasal ameloblastoma confined to the nasal cavity include the nasal septum, lateral nasal wall and turbinates. ${ }^{4,8,10}$ Among the paranasal sinuses, the maxillary sinus was most commonly affected, followed by the ethmoid, frontal and sphenoid sinuses (the latter with only one reported case). ${ }^{8}$ In this case, the mass was surgically confirmed to arise from the postero-medial choana with extension into the sphenoid sinus, an occurrence not previously reported.

Gnathic ameloblastoma arises most frequently from rests of primitive dental lamina in the gingiva, alveolar bone above the level of tooth apices, follicular walls of unerupted teeth, lining of odontogenic cysts, and even gingival surface epithelium. ${ }^{10}$ Controversy surrounds the origin of extragnathic ameloblastoma particularly those arising in the nasal cavity. This entity is believed to arise from the pluripotential 


\section{CASE REPORTS}

basal layer of the surface epithelium or from ectopic epithelial rests. ${ }^{8,10}$

Management of extragnathic sinonasal ameloblastoma as with ameloblastoma is surgical with good prognosis following complete tumor excision. The approach depends on the extent of the tumor and experience of the surgeon. The goal is to completely remove the entire tumor to reduce the risk of recurrence with preservation of as much normal tissue as possible to reduce morbidity. In the current case, a lateral rhinotomy approach was employed due to the extensive involvement of adjacent structures by tumor. Recurrences have been documented even after adequate surgery, and close follow up should be emphasized.

Extragnathic sinonasal ameloblastoma, an extremely rare variant of ameloblastoma, is benign but may present as a locally aggressive entity, mimicking malignant tumors involving the nasal cavity and/or the paranasal sinuses. Diagnosis is primarily based on histopathology but radiologic and intraoperative findings aid in its distinction from the closest differentials. Apart from CT scan as a primary imaging modality, MRI plays a crucial role in extensive cases where involvement of vital structures needs to be assessed. Complete surgical excision remains the treatment of choice, and coupled with good follow up should serve to improve the prognosis of patients.

\section{ACKNOWLEDGEMENTS}

The author thanks Dr. Arsenio Claro A. Cabungcal and Dr. Jan Warren A. Holgado of the Department of Otorhinolaryngology, Philippine General Hospital, University of the Philippines Manila for their valuable contributions; the former, in managing the patient in this report and the latter, in helping draft the initial manuscript.

\section{REFERENCES}

1. Angadi PV. Head and Neck: Odontogenic tumor: Ameloblastoma [monograph on the Internet] Atlas Genet Cytogenet Oncol Haematol; May 2010 [cited 2012 Feb 8]. Available from: http:// AtlasGeneticsOncology.org/Tumors/AmeloblastomID5945.html

2. Guilemany JM, Ballesteros F, Alós L, Alobid I, Prades E, Menéndez LM, Cardesa A. Plexiform ameloblastoma presenting as a sinonasal tumor. Eur Arch Otorhinolaryngol [serial on the Internet]. 2004 Jul [cited 9 Feb 2012];261(6):304-306. Available from: http://www.deepdyve. $\mathrm{com} / \mathrm{lp} /$ springer-journals/plexiform-ameloblastoma-presenting-as-a-sinonasal-tumore32PtQ004D

3. Leong SC, Karkos PD, Krajacevic J, Islam R, Kent SE. Ameloblastoma of the sinonasal tract: A case report. Ear Nose Throat J [serial on the Internet]. 2010 Feb;89(2):70-71. Available from: http:// www.mdlinx.com/otolaryngology/news-article.cfm/3353607/sinonasal

4. Ereño C, Etxegarai L, Corral M, Basurko JM, Bilbao FJ, López Jl. Primary sinonasal ameloblastoma [abstract]. APMIS 2005 Feb [cited 9 Feb 2012];113(2):148-150. Available from http://onlinelibrary. wiley.com/doi/10.1111/j.1600-0463.2005.apm1130210.x/abstract

5. Ang MA, Vergel de Dios A, Carnate J. Primary sinonasal ameloblastoma in a Filipino female. Philipp J Otolaryngol Head Neck Surg [serial on the Internet]. 2011 Jul-Dec [cited 8 Feb 2012];26 (2):39-41. Available from: http://www.pso-hns.org/psojournals/pjohns/2011b/08-under microscope.pdf

6. Sharma V, Purohit JP. Ameloblastoma: a rare nasal polyp. Indian J Otolaryngol Head Neck Surg [serial on the Internet]. 2011 Jul [cited 8 Feb 2012]; 63(Suppl 1):893-895. Available from http:// www.ncbi.nlm.nih.gov/pmc/articles/PMC3146654/pdf/12070_2011_Article_211.pdf

7. Michaels L, Hellquist H. Ear, nose and throat histopathology. $2^{\text {nd }}$ ed. London: Springer; 2001. 254-255.

8. Schafer DR, Thompson LDR, Smith BC, Wenig BM. Primary ameloblastoma of the sinonasal tract: a clinicopathologic study of 24 cases. Cancer [serial on the Internet]. 1998 Feb [cited 9 Feb 2012]; 82(4):667-674. Available from http://www.lester-thompson.com/articles/Cancer/Cancer1998-02_Primary ameloblastoma of the sinonasal tract.pdf

9. Deutsch H, Kothbauer K, Persky M, Epstein FJ, Jallo Gl. Infrasellar craniopharyngiomas: case report and review of the literature. Skull Base [serial on the Internet]. 2001 May [cited 8 Feb 2012];11(2):121-128. Available from: http://www.ncbi.nlm.nih.gov/pmc/articles/PMC1656789/ pdf/skullbase00006-0037.pdf

10.Gnepp DR. Diagnostic Surgical Pathology of the Head and Neck. 2nd ed. Philadelphia, PA: Saunders; 2009. 168-170.

11.Kruze AL, Zwahlen RA, Gratz KW. New classification of maxillary ameloblastic carcinoma based on an evidenced-based literature review over the last 60 years. Head Neck Oncol [serial on the Internet]. 2009 Aug 12 [cited 23 Feb 2012];1(1):31. Available from: http://www. headandneckoncology.org/content/pdf/1758-3284-1-31.pdf

12.Prashad KV, Ramesh V, Balamurali PD, Premalatha B. Ameloblastic carcinoma-a case report highlighting its variations in histology. J. Int Oral Health [serial on the Internet]. 2011 December [cited 24 May 2013\};3(6):37-42. Available from: http://www.ispcd.org/ cmsdev/userfiles/ rishabh/JIOH-03-06-037.pdf 